\title{
Case: Causation vs. correlation: Naturopathic medicine vs. natural history of a disease
}

\author{
Roshan Navaratnam ${ }^{1}$; Hassan Razvi ${ }^{2,3}$; Nicholas Power ${ }^{2,3}$ \\ ${ }^{1}$ Schulich School of Medicine; ${ }^{2}$ Department of Surgery (Urology); ${ }^{3}$ Department of Surgical Oncology; Western \\ University, London, ON, Canada
}

Cite as: Can Urol Assoc J 2018 June 8; Epub ahead of print. http://dx.doi.org/10.5489/cuaj.5058

Published online June 8, 2018

$* * *$

\section{Introduction}

Urothelial carcinoma (UC) is the fourth most common type of cancer and arises from the urogenital epithelial. Between $5-10 \%$ of primary UC originate from renal pelvis and ureters and are collectively called upper tract urothelial carcinoma (UTUC). The estimated annual incidence of UTUC in Canada is 1-2/ 100,000. ${ }^{1}$ It most commonly affects patients in the sixth and seventh decade of life with a male predominance. In developed countries, tobacco smoking is the greatest risk factor. UTUC is often multifocal as the entire urothelial surface is affected by the same carcinogen. $^{2}$

The gold standard treatment of localized UTUC is a radical nephroureterectomy due to the frequent occurrence of synchronous or metachronous tumours. For patients with high risk disease (ie. T3-T4 and/or patients with positive lymph nodes) adjuvant chemotherapy is also suggested $^{2}$. Post-operative recurrences are common. In general, bladder recurrence occurs in 22$47 \%$ while extravesical recurrences occur in $0-12 \%$ of patients with UTUC. ${ }^{1}$

Naturopathic treatments have frequently been mentioned in the context of "curing" cancer. However, there are limited studies that follow up on these numerous treatment options and the studies that are available often are unreliable or invalid. ${ }^{3}$ Therefore, many of these rare cases are generally attributed to the spontaneous regression of cancer.

We present a case of a patient with UTUC who had a local recurrence following definitive therapy and experienced resolution of cancer following a naturopathic treatment option.

\section{Case report}

A 65-year-old male had a left nephroureterectomy for a high grade pT3 urothelial carcinoma of the left renal pelvis in 2001. Surveillance CT done in December 2002 and March 2003 revealed para-aortic lymphadenopathy that was enlarging over time. A CT guided biopsy in April 2003 was found to be positive for malignancy, consistent with UC infiltration of a lymph node. 
However, the patient refused to have subsequent chemotherapy and instead chose a naturopathic option that he had learnt about through word of mouth known as the Bruess Cancer Cure.

Following this diet, he had a CT scan in May 2004, that showed the para-aortic nodule to be resolved. He had subsequent surveillance imaging with regular cystoscopies until March 2013 which were stable with no evidence of recurrent disease. He also subsequent CT scans and an MRI in 2017 related to a large bowel obstruction that further confirmed no evidence of recurrent disease.

\section{Discussion}

The first documented case of the "healing power of nature" was by Hippocrates, over 2400 years ago who believed in the body to heal itself with the help of nature. Since then, naturopathic medicine has broadened its scope to include the use of botanical medicine, clinical nutrition, hydrotherapy, homeopathy, naturopathic manipulation and more. Following a decline in favourability in the 1900s, there has been a recent resurgence in naturopathic medicine with the public desire for greater control in their health and dissatisfaction with "allopathic” medicine.

One of the most famous Canadian cases supporting claim to naturopathic medicine is the story of Ronnie Hawkins, a famous Canadian musician who was clinically diagnosed with terminal pancreatic cancer in 2002 by his surgeon, despite negative biopsies. After refusing chemotherapy and trying various forms of alternative treatments, Ronnie accepted the help of an adolescent child from British Columbia who was believed to have 'telepathic healing powers' Following several treatments from this child, an MRI in 2003, confirmed by his surgeon, suggested Ronnie was cancer free. ${ }^{4}$

This case report, in particular, describes a 65-year-old male with metastatic recurrence of UCUT proven by a biopsy, which had later resolved without conventional medical treatment.

The Bruess Cancer Cure consists of a 42 day fast in combination with "special” vegetable juices and teas. The evidence behind this diet is poor with only one inadequately designed clinical trial conducted without the use of control groups and proper exclusion criteria. ${ }^{5}$ The trial showed two complete remissions but three deaths in eight patients with metastatic disease. An alternative explanation for the disappearance of the patient's metastatic cancer is spontaneous regression. There are case reports describing regression of multiple pulmonary metastases from UTUC. ${ }^{6}$ This phenomenon is believed to be secondary to immune system activation, angiogenesis and matrix metallo-proteinase activation. ${ }^{7}$

With growing popularity in Complementary and Alternative Medicine (CAM), this has become a common interest in patients seeking different forms of cancer treatment. CAM has been defined as "a group of medical and healthcare systems, practices and products that are not presently apart of conventional medicine" and can be used in addition to conventional treatment (complementary) or as a replacement (alternative). This definition includes many treatment options with varying origins and levels of evidence. Because of this, the Canadian Medical Association's (CMA) position on CAM is that it should not be recommended by physicians and publically funded until they are supported by scientifically-valid evidence. However, they do 
recommend patients to report use of all health products so that physicians can have a discussion with patients about the reliability of these treatment options and potential harm associated with concomitant conventional treatment. ${ }^{8}$

It is important to take this a step further by first understanding why patients are turning to CAM as a treatment option. This gain in popularity is a result of combination of factors including: curiosity, distrust and dissatisfaction of conventional treatments, and belief in a "holistic" approach to health. Better communications with patients can be important in identifying motivation to use CAM, improving patient-physician relationship, and assisting with appropriate use of CAM, and reducing any dissatisfaction with oncology medicine. ${ }^{8}$ In the most optimal situations, a careful balance can be attained with evidence based medicine and the comfort from the best of CAM options.

Recently, there has been a rise in the integrative oncology centres across Canada. The aim behind these centers is to provide a comprehensive approach to patient care by treating cancer patients physically, mentally and emotionally. There is a collaborative approach with patient, families, family physicians, oncologist, surgeons and naturopathic doctors to optimize overall patient wellness. This is an interesting approach as it can incorporate patient preferences for CAM but also incorporate a safe approach to cancer treatment. Furthermore, research has shown that a number of complementary therapies can help lessen treatment side effects such as nausea or fatigue, as well as cope with stress and anxiety that patients may face during their cancer journey. $^{9}$

\section{Conclusion}

With a lack of evidence behind the Bruess Cancer Cure, it is difficult to accept its efficacy as a treatment for cancer. An alternative explanation to the resolution of the patient's cancer is spontaneous regression. Although rare, there have been documented case reports in literature for various cancers, including metastatic UCUT. Despite this lack of evidence for naturopathic treatments, it is important to be aware that these options have gained popularity in providing comfort and relief to patients. Therefore, if the patient is determined to consider these options, it should be safely used in conjunction with any traditional treatment. 


\section{References}

1. Roupret M, Babjuk M, Comperat E, Zigeuner R, Sylvester R, Burger M, et al. European guidelines on upper tract urothelial carcinomas: 2013 update. European Urology. 2013. 63 (6): 1059-1071.

2. Kirkali Z, Tuzel E. Transitional cell carcinoma of the ureter and renal pelvis. Critical Reviews in Oncology/Hematology. 2003. 47: 144-169.

3. Cassileth, BR. Alternative and Complementary Cancer Treatments. The Oncologist.1996. 1(3): 173-179.

4. Hampson, S. Cancer-free, he’s rompin’ again. The Globe and Mail. 2003. Retrieved from: https://www.theglobeandmail.com/arts/cancer-free-hes-rompinagain/article1014493/?page=all

5. Krumwiede K, Horneber M, CAM-Cancer Consortium. Bruess Cancer Cure. CAMCancer: Complementary and Alternative Medicine for Cancer. Updated: February 2017.

6. Lome LG, Navani S, Aral IM. Spontaneous Regression of Pulmonary Metasases from Transitional Cell Carcinoma of the Bladder. Cancer. 1970. 25: 415-418.

7. Jessey T. Immunity over inability: The Spontaneous Regression of Cancer. Journal of Natural Science, Biology and Medicine. 2011. 2 (1): 43-49.

8. Canadian Medical Association. CMA Policy: Complementary and Alternative Medicine 2015 Update. The Association. 2015.

9. Canadian Cancer Society. Complementary Therapies. 2018 Canadian Cancer Society. Retrieved from: http://www.cancer.ca/en/cancer-information/diagnosis-andtreatment/complementary-therapies/?region=bc

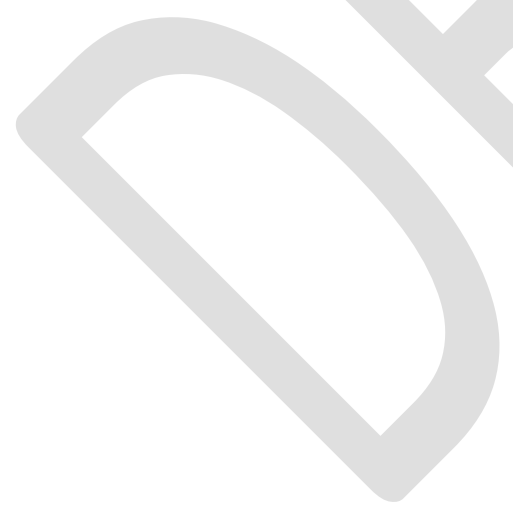

